\title{
Comparison of a New IgG-EIA for the Detection of Anti-Plasmodium Antibodies with Two Currently Used Assays
}

\author{
Christoph Niederhauser $^{a, b, c}$ Caroline Tinguely ${ }^{a}$ Jens Dreier ${ }^{d}$ \\ Tanja Vollmer $^{d}$ Hans Peter Marti ${ }^{e}{ }^{f} \quad$ Beatrice Nickel ${ }^{e}, f \quad$ Julia Maria Klemens ${ }^{g}$ \\ Jens Miguel Warnecke ${ }^{g}$ Peter Gowland ${ }^{a}$ \\ anterregional Blood Transfusion SRC, Bern, Switzerland; 'b Institute for Infectious Diseases, Faculty of \\ Medicine, University of Bern, Bern, Switzerland; ${ }^{c}$ Faculté de Biologie et de Médecine, Université de Lausanne, \\ Lausanne, Switzerland; 'Institut für Laboratoriums- und Transfusionsmedizin, Herz- und Diabeteszentrum \\ Nordrhein-Westfalen, Universitätsklinik der Ruhr-Universität Bochum, Bad Oeynhausen, Germany; \\ eSwiss Tropical and Public Health Institute, Basel, Switzerland; fUniversity of Basel, Basel, Switzerland; \\ Institute for Experimental Immunology, EUROIMMUN Medizinische Labordiagnostika AG, Lübeck, Germany
}

Keywords

ElA · Antibody detection · Plasmodium spp.

\begin{abstract}
Background: Malaria is a mosquito-borne infectious disease caused by protozoan parasites of the genus Plasmodium. As migration of populations from endemic areas to Europe and overseas recreational travel to endemic regions increase, there is also a growing risk of transfusion-transmitted tropical diseases by blood components. Material and Methods: In the present study two routine Plasmodium spp. ELISA (CAPTIA ${ }^{\mathrm{TM}}$ Malaria EIA, Trinity Biotech, and Malaria EIA, BioRad) were compared with a new commercial ELISA (ELISA IgG, EUROIMMUN). From December 1, 2015 until November $30,2016,1,096$ plasma samples from blood donors with a potential risk of malaria infection were collected at two blood transfusion centres in Germany and Switzerland. $R \boldsymbol{e}$ sults: The samples were tested comparatively with the ELISA from EUROIMMUN and the routine test used at the respective centre. Thirty-four of 595 (5.7\%) tested blood samples from centre 1 and 49 of 501 (9.8\%) tested blood samples from centre 2 showed reactivity on either or both ELISAs. All 83 reactive samples were sent for confirmation to the Diagnostic Centre of the Swiss Tropical and Public Health Institute (Swiss TPH) in Basel, Switzerland. Sixteen samples,
\end{abstract}

which previously were reactive in the routine Plasmodium spp. ElA assays, were proven positive after confirmation testing (i.e., 4 positive and 12 inconclusive results), indicating an anti-Plasmodium antibody prevalence in blood donations of $1.5 \%$. From these 16 reactive samples, 13 were also detected by the index test, resulting in an assay sensitivity of $81.2 \%$. A specificity of $98.6 \%$ was calculated $(1,065 / 1,080$ confirmed negative samples). The overall agreement with the reference centre was $95.8 \%$ in centre 1 and $94 \%$ in centre 2. Conclusion: The comparison of the new EUROIMMUN ELISA and the established CAPTIA ${ }^{\text {TM }}$ Malaria EIA (Trinity Biotech) and Malaria EIA (BioRad) used for routine blood donor screening in two laboratory blood donation centres revealed that all tested ELISAs show comparable sensitivities and are equally suitable for anti-Plasmodium antibody screening in blood banks.

(c) 2021 The Author(s)

Published by S. Karger AG, Basel

\section{Introduction}

Four different Plasmodium species are relevant for human infections: $P$. falciparum, $P$. vivax, $P$. ovale and $P$. malariae. Recently, P. knowlesi has emerged as an additional human pathogenic species. Though it is primarily zoonotic, infecting macaque monkeys, recently different

\section{karger@karger.com} www.karger.com/tmh

Karger ${ }^{\prime \prime} \%$

BOPEN ACCESS
(C) 2021 The Author(s)

Published by S. Karger AG, Base

This article is licensed under the Creative Commons Attribution 4.0 International License (CC BY) (http://www.karger.com/Services/ OpenAccessLicense). Usage, derivative works and distribution are permitted provided that proper credit is given to the author and the original publisher.
Correspondence to:

Christoph Niederhauser, christoph.niederhauser@itransfusion.ch 
human populations in South-East Asia, as well as travellers returning from endemic regions, were found to be infected. P. knowlesi was previously not recognized as a human pathogen and was thus probably misdiagnosed as the more benign and morphologically similar species $P$. malariae [1].

According to a World Health Organisation (WHO) report, 228 million new malaria infections occurred in 2018; of which approximately 405,000 were fatal. Transfusion of contaminated blood components is known to be a possible mode of human-to-human transmission. The incidence of transfusion-transmitted malaria (TTM) is particularly high in endemic regions, but there is also an increasing risk in non-endemic countries. This is primarily due to the increased number of migrants from endemic areas, as well as a rise in the number of travellers visiting endemic countries. Indeed, numerous cases of TTM from around the globe have been reported during the last decade (e.g., Brazil, Italy, Canada, Switzerland, the UK, Malaysia, and France) [2-8]. Although rare, TTM poses a risk to blood transfusion services worldwide, particularly since TTM cases are the consequence of infections from "semi-immune" donors who do not show clinical symptoms and who often have undetectable levels of malaria parasites circulating in their blood [4, 5, 8-11].

According to the current European regulations, it is mandatory for blood donors who present with risk of malaria to be deferred for 6 months up to 4 years, depending on the severity of their exposure risk to the Plasmodium parasite. Furthermore, during the last 2 decades many transfusion centres in western countries, including France, the UK, Australia, Denmark, Finland, New Zealand, and Switzerland, and partly volunteer-based centres in Germany, have implemented selective malaria antibody screening programmes to identify donors with previous malaria infection $[5,12-16]$.

Blood smear tests, RDTs, and PCRs are not suitable for screening of potentially infected blood donors and thus ELISA-based tests were developed to test for specific antiPlasmodium antibodies in blood donations $[17,18]$. These serological tests often apply parasite antigens from non-sexual blood stages, which are the main target of the immune response. Therefore, Plasmodium-specific antibodies are detected at the earliest 1 or 2 weeks after the initial infection [19]. Most of the commercially available ELISAs use antigens derived from $P$. falciparum and $P$. vivax. The detection of antibodies against other malaria species (e.g., P. ovale, P. malariae, and P. knowlesi) thus relies solely on cross-reactivity of antibodies to the $P$. falciparum and $P$. vivax antigens. Although in most cases there are indeed cross-reactive antigens to these antigens, there are reports showing reduced sensitivity for the detection of antibodies generated against the other malaria species [14]. To combat this discrepancy, several recently developed ELISA tests have begun to include further antigens from P. ovale and P. malariae [18]. So far, however, the detection of specific antibodies against $P$. knowlesi has not been included in any blood donor-screening ELISA.

Here, we report a prospective study evaluating the serological screening of blood donors originating from malaria-endemic regions and travellers returning from such regions for anti-Plasmodium spp. antibodies to assess the performance of the first commercial ELISA (EUROIMMUN EIA) using recombinant antigens for all 5 human pathogenic Plasmodium species. This ELISA was compared to 2 established Plasmodium species ELISA tests used in routine blood donor screening at two different laboratory centres. Confirmatory testing of reactive samples was conducted at the Swiss National Reference Centre for Imported Parasitic Diseases at the Diagnostic Centre of the Swiss Tropical and Public Health Institute (Swiss TPH) in Basel, Switzerland.

\section{Materials and Methods}

\section{Sample Origin and Collection Strategy}

Plasma samples were collected prospectively from December 1, 2015 until November 30, 2016. The samples were selected from adult blood donors ( $\geq 18$ years) who had reported visiting a malaria-endemic region since their previous donation or were born and/ or lived in a malaria-endemic region for more than 6 months. The study was conducted at two blood transfusion laboratories: the Institute for Laboratory and Transfusion Medicine, Bad Oeynhausen (centre 1, Germany), where 612 plasma samples were collected (collective A), and the Interregional Blood Transfusion Centre SRC, Bern (centre 2, Switzerland), where 502 samples were collected (collective B).

\section{Testing Strategy}

Study samples were analysed by the two respective centres using the screening Malaria ELISA routinely applied at that centre. Additionally, both centres analysed the samples with the novel Anti-Plasmodium ELISA IgG (EUROIMMUN, Germany). Those samples reactive for anti-Plasmodium IgG in the corresponding screening ELISA or the EUROIMMUN index ELISA were sent to the Swiss TPH for confirmation. Negative samples in the screening ELISA and the index test were not forwarded for confirmation. Samples above the cut-off with the CAPTIA ${ }^{\mathrm{TM}}$ Malaria EIA, Trinity Biotech (centre 1), and with the Malaria EIA, BioRad (centre 2) were considered reactive in concordance with the routine procedures at the blood donation centres. For the purpose of this study, reactive samples and samples in the borderline range between the ratio of 0.8 and 1.1 using the index ELISA from EUROIMMUN were also considered reactive and thus sent for confirmation. All inconclusive and positive results determined at the Swiss TPH were considered confirmed reactive.

Index Test: Anti-Plasmodium ELISA IgG, EUROIMMUN

The index ELISA was developed to qualitatively and semiquantitatively detect antibodies in human serum or plasma to all 5 pathogenic Plasmodium species (P. falciparum, P. vivax, P. ovale, $P$. malariae, and $P$. knowlesi). The test uses microtitre plates coated with a mixture of recombinant target antigens from all 5 species. Specific antibody binding is visualised by the addition of an anti- 
Fig. 1. Algorithm for the comparison of the two screening assays with the EUROIMMUN index assay.

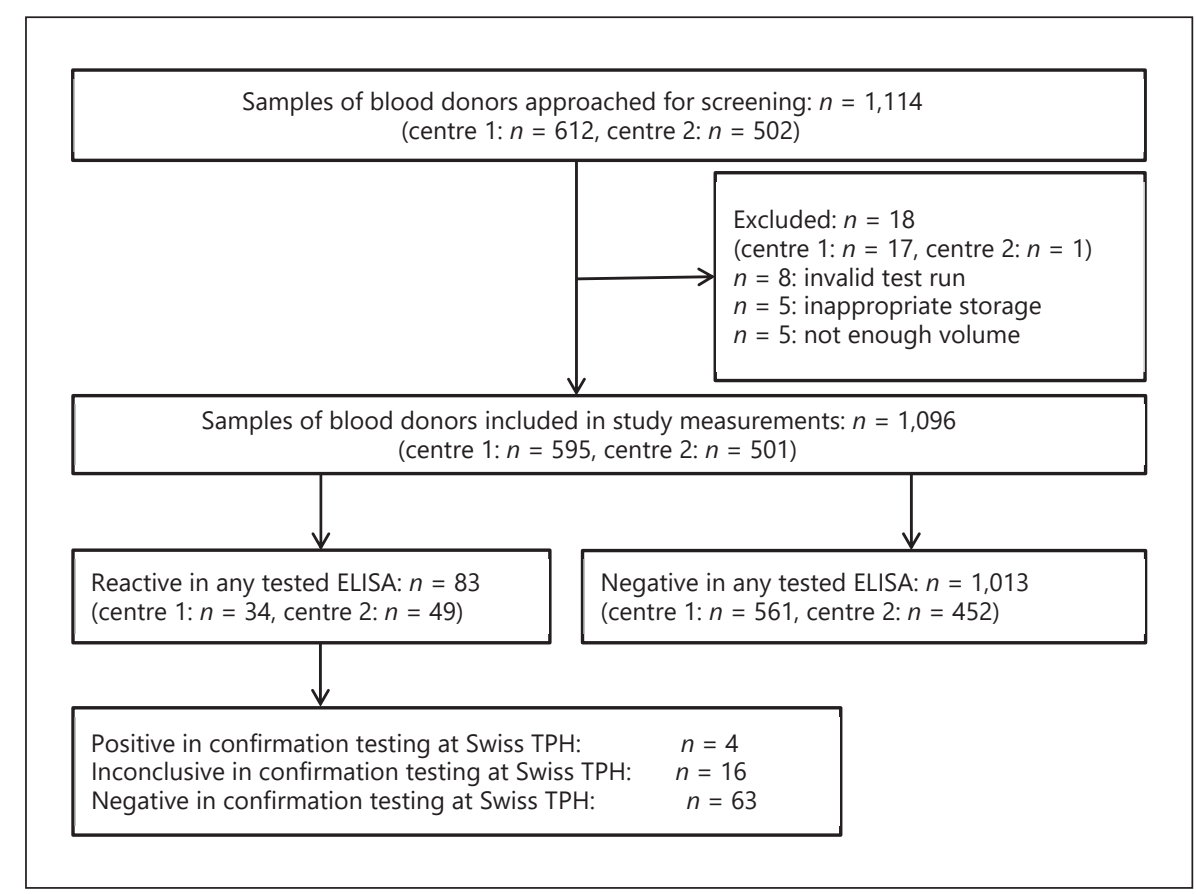

human IgG conjugated to peroxidase, which catalyses the colour change of the chromogenic substrate $\mathrm{TMB} / \mathrm{H}_{2} \mathrm{O}_{2}$. The ELISA was conducted according to the manufacturer's instructions and the results are reported as the extinction ratio of sample to calibrator. Samples are positive with a ratio $\geq 1.1$. A borderline range is defined for a ratio between 0.8 and 1.1. Within this study, samples with borderline or positive results were classified as reactive.

Screening Tests: CAPTIA ${ }^{T M}$ Malaria EIA, Trinity Biotech

(Centre 1) and Malaria EIA, BioRad (Centre 2)

According to the test instructions, both kits are manufactured by Trinity Biotech and use exactly the same antigens for detection of total anti-malaria antibodies. Therefore, we consolidated both assays under the term "screening ELISA." Both ELISAs detect qualitatively and semi-quantitatively antibodies against $P$. falciparum, $P$. vivax, $P$. ovale, and $P$. malariae in human serum or plasma. The microtitre plates are coated with total protein extract from $P$. falciparum and a recombinant antigen from $P$. vivax. Due to antigenic similarity between the Plasmodium species $P$. ovale and $P$. malariae, the manufacturer reports that antibodies to these species are detected as well with this assay. The total malaria antibody binding from donor samples is visualised by addition of the same antigens conjugated to horseradish peroxidase. Both ELISAs were conducted according to manufacturer's instructions. The cut-off was set as the mean of the negative controls plus 0.100 optical density units; no borderline range was defined in routine use. In this study, all samples above the cut-off were considered reactive.

\section{Reference Test: Malaria ELISA and IFAT}

The reference strategy chosen for this study is that used routinely for malaria antibody testing in all Swiss blood donation centres and follows the algorithm described in the national guidelines of the Blood Transfusion Service of the Swiss Red Cross [20]. Briefly, it states that all risk donations must be screened by an additional independent ELISA or IFAT (indirect immunofluorescence test). Those donations with a negative result for Plasmodium spp. antibodies are regarded as free from Plasmodium and all blood products from this donation can be released for transfusion. All samples reactive in the screening ELISAs at the test centre were sent for confirmation to the Swiss TPH with their in-house ELISA and IFAT that use antigens of cultured P. falciparum erythrocytic stages. The in-house ELISA in combination with the IFAT exhibits a sensitivity of $>99 \%$ for $P$. falciparum, $P$. vivax, $P$. malariae, $P$. ovale, and $P$. knowlesi antibodies for blood samples taken $>1$ week after the first fever episode. Sensitivity is considerably reduced for blood samples taken $<1$ week after the onset of symptoms for infections with all Plasmodium spp. due to lack of seroconversion (see online suppl. Table 1; for all online suppl. material, see www. karger.com/doi/10.1159/000515842). The specificity of the combined test is $>99 \%$ for healthy donors. Cross-reactivity with infections of other blood protozoa species cannot completely be excluded.

\section{P. falciparum spp. ELISA}

Malaria serology was performed with an in-house screening ELISA for detection of specific Plasmodium spp. antibodies. P. falciparum antigen (strain NF54) from erythrocytic stages was coated in $0.05 \mathrm{M}$ sodium carbonate buffer, $\mathrm{pH} 9.6$, to Immulon $2 \mathrm{HB}$ plates (ThermoScientific, Wohlen, Switzerland). After washing, diluted plasma samples were added to the plates and incubated for $15 \mathrm{~min}$ at $37^{\circ} \mathrm{C}$. After additional washing steps, horseradish peroxidase conjugated goat-anti-human IgG (KPL, 474-1006, BioConcept Ltd, Allschwil, Switzerland) was added. Plates were incubated for 15 min at $37^{\circ} \mathrm{C}$, subsequently washed, and o-Phenylendiamine Dihydrochloride (OPD, Sigma, Buchs, Switzerland) was added. The reaction was stopped with $8 \mathrm{M} \mathrm{H}_{2} \mathrm{SO}_{4}$, and absorption was read with a Multiscan FC reader (ThermoScientific) at $492 \mathrm{~nm}$. The cutoff OD for the ELISA was $<0.15$, inconclusive range OD was $0.15-$ 0.29 , and positive OD was $\geq 0.30$. All plasma samples giving positive or equivocal results were additionally tested with an in-house confirmatory malaria IFAT.

\section{Malaria IFAT Test}

Immunofluorescence staining was performed with cultured erythrocytic stages of $P$. falciparum (strain NF54) mounted on glass slides. Slides were stored at $-80^{\circ} \mathrm{C}$ until the day of use. For 
Table 1. Comparison of data from centre 1

\begin{tabular}{llll}
\hline $\begin{array}{l}\text { Centre 1 } \\
(n=595)\end{array}$ & Trinity Biotech Screening \\
\hline EUROIMMUN & & \\
Index & Reactive & 9 & Negative \\
& Negative & 2 & 23 \\
& & & 561 \\
\hline
\end{tabular}

Table 2. Comparison of data from centre 2

\begin{tabular}{|c|c|c|c|}
\hline $\begin{array}{l}\text { Centre 2 } \\
(n=501)\end{array}$ & BioRad S & & \\
\hline \multirow{3}{*}{$\begin{array}{l}\text { EUROIMMUN } \\
\text { Index }\end{array}$} & & Reactive & Negative \\
\hline & Reactive & 19 & 18 \\
\hline & Negative & 12 & 452 \\
\hline
\end{tabular}

IFAT analysis, the slides were quickly air dried at room temperature and then fixed with acetone. Plasma samples were diluted in PBS pH 7.2 and applied to the slide slots. Three control samples were present on each slide: 1 positive, 1 equivocal, and 1 negative control. After $25 \mathrm{~min}$ of incubation at $37^{\circ} \mathrm{C}$ in a wet chamber, the slides were washed with PBS pH 7.2 and air dried. FITC conjugated $\mathrm{F}(\mathrm{ab})$ '2 2 anti-IgG/A/M (BioRad, No. 30244) diluted in $0.01 \%$ Evans blue in PBS was added and the slides were incubated for 25 min at $37^{\circ} \mathrm{C}$, washed, dried, and a cover glass was mounted with buffered glycerol. Slides were examined immediately with a fluorescence microscope. The IFAT exhibits a sensitivity of $>99 \%$ for Plasmodium spp. and a specificity of $>99 \%$ for healthy blood donor samples (see the appendix of online suppl. Table 1)

The results of the combined ELISA and IFAT confirmation tests are reported as positive, inconclusive, or negative. If these confirmation tests are negative, then the blood donation is consid ered free of malaria and the corresponding blood products are released for transfusion. Those samples classified as positive or inconclusive are considered to pose a risk of transmitting Plasmodium via blood transfusion, even if this represents an antibody titre from a previous infection, and the blood products are destroyed. These donors are deferred for at least 3 years due to safety reasons.

\section{Results}

\section{Comparison of Performance of Screening and Index} ELISAs

During the 1-year study period, 1,114 samples were screened in both test centres. After the exclusion of 18 samples for various reasons ( 17 from centre 1 and 1 from centre 2), 1,096 samples were included in the study. Figure 1 shows the sample distribution by the two study sites and the flow of patient samples. In total, 83 of 1,096 reactive samples $(7.6 \%)$ were identified in the screening or index ELISAs. The number of reactive samples identified at the two centres was: 34 out of $595(5.7 \%)$ at centre 1 , and 49 out of $501(9.8 \%)$ at centre 2.
At centre $1(n=595), 11$ samples were reactive in the screening ELISA while 32 samples were reactive in the index test (Table 1). Discrepant results were obtained for 25 samples, whereas only 9 samples were consistently reactive and 561 samples were negative by both tests. At centre $2(n=501), 31$ samples were reactive with the screening ELISA and 37 samples were reactive with the index ELISA. From these 49 reactive samples, 30 were reactive with either the index or screening ELISA, whereas 19 samples were reactive in both assays. The remaining 452 samples were negative (Table 2). The overall concordance of $95.8 \%$ was determined at centre 1 , and $94.0 \%$ at centre 2 .

\section{Confirmation at the Reference Centre}

All 83 samples reactive in the screening or index antiPlasmodium ELISAs were sent to the Swiss TPH for confirmation. From the 28 samples which were reactive in both the index and screening ELISAs, 4 were confirmed positive, 9 cases showed an inconclusive result, and a further 15 samples were classified as negative (Fig. 2). None of the 14 samples reactive in the screening but negative in index ELISA were confirmed positive; however, 3 samples yielded an inconclusive result and 11 were negative. Likewise, none of the 41 samples initially negative in the screening ELISA but positive in the index test were confirmed positive at the reference centre; however, 4 samples showed an inconclusive result and a further 37 samples were negative. Thus, following the reference strategy, from the 42 samples which were reactive in the screening ELISAs, 16 samples were declared positive (4 positive and 12 inconclusive samples) by the Swiss TPH.

Calculations of prevalence, sensitivity, and specificity of the index EUROIMMUN anti-Plasmodium ELISA (IgG) test were performed against the reference strategy. As 17 samples were finally confirmed positive or inconclusive after confirmation testing (i.e., 4 positive and 13 inconclusive samples from 1,096), an anti-Plasmodium antibody prevalence in blood donations of $1.55 \%$ was calculated. Of the 20 confirmed reactive samples, 17 samples were detected by the index test, resulting in an assay sensitivity of $85.0 \%$. The specificity of $95.2 \%$ was calculated (1,024 out of 1,076 confirmed negative samples).

\section{Discussion}

In non-endemic countries, the risk of TTM is often linked to asymptomatic infections acquired during previous residency or travel activities in endemic regions combined with semi-immunity. Selective screening of blood donors with risk for infections with Plasmodium spp. using serological assays has demonstrated to be a feasible and cost-effective strategy to minimize the number of re- 


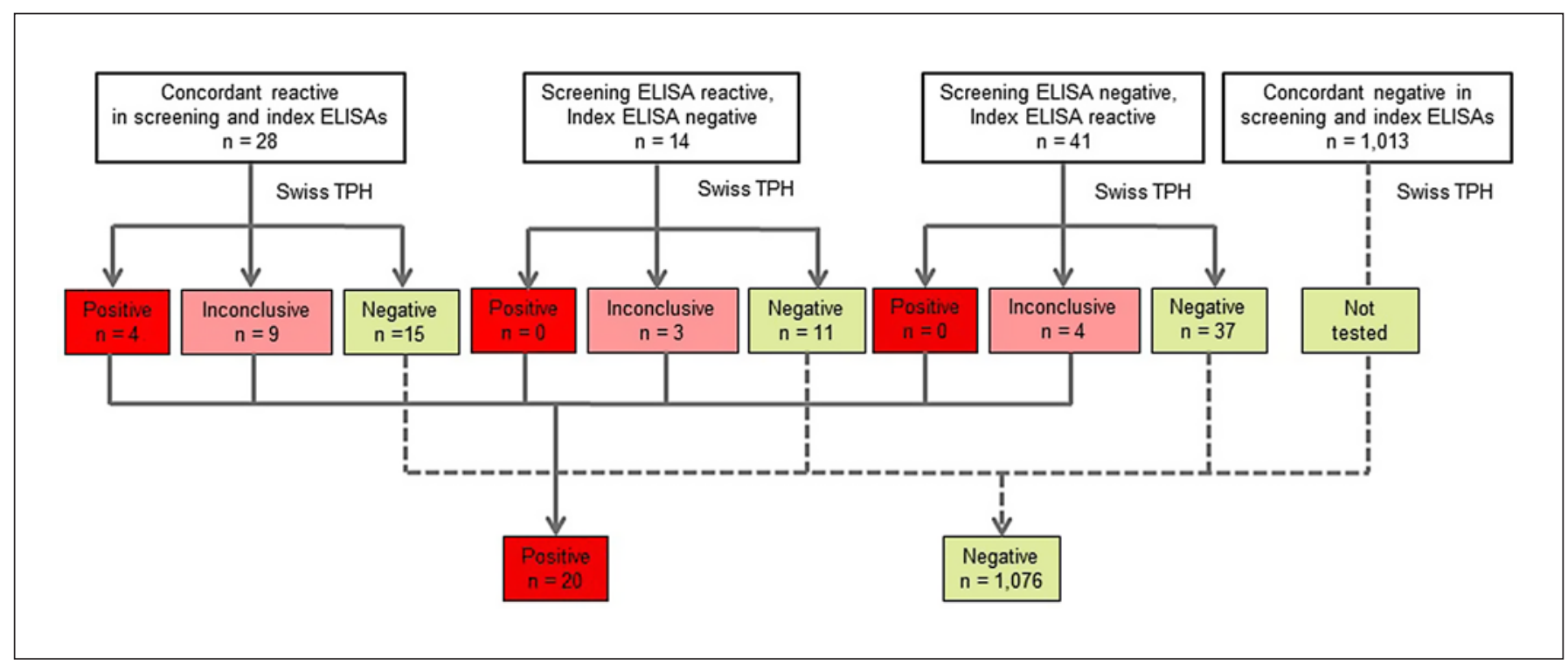

Fig. 2. Further analysis of reactive samples from the screening and the EUROIMMUN index assays at the National Reference Centre for Imported Parasitic Diseases at the Swiss TPH.

jected blood donations but keep a high blood safety $[5,7$, $13,14,17,21,22]$.

In this study, the novel EUROIMMUN anti-Plasmodium ELISA (IgG) was compared to the current screening ELISAs routinely used at two independent blood transfusion centres in order to test blood donors at risk for having acquired malaria. The new anti-Plasmodium ELISA (IgG) exhibited a high concordance to the routine screening test at both centres (95.8\%: centre 1, and 94.0\%: centre 2 ). The relative discrepancy of $5.0 \%$ between the screening and index results may be attributed to the different antigenic substrates used in the different ELISA tests. Furthermore, the screening ELISA produced by Trinity Biotech uses protein extract from $P$. falciparum and a recombinant antigen from P. vivax, whereas the anti-Plasmodium ELISA (IgG) from EUROIMMUN applies recombinant antigens from all 5 human pathogenic Plasmodium species. Moreover, a borderline range is defined for the results obtained with the anti-Plasmodium ELISA (IgG). In favour of a high blood safety level, all the borderline results were considered reactive.

Being in line with the idea of a two-step reference strategy, only a subset of reactive results obtained with the screening test were confirmed positive at the reference centre ( 4 of 42). All 4 samples were also found to be reactive by both the EUROIMMUN Index and ELISA and Trinity Biotech screening ELISAs.

Sensitivity and specificity calculations for the index EUROIMMUN test were performed against the reference strategy yielding 85.0 and $95.2 \%$, respectively. All 4 samples reactive with the EUROIMMUN ELISA were confirmed reactive at the Swiss TPH but were negative with the Trinity Biotech ELISA. Likewise, 3 samples reactive with the screening ELISA were confirmed at the Swiss TPH but were negative with the index ELISA. The Trinity Biotech screening ELISA thus achieved a sensitivity of $80.0 \%$ ( 16 out of 20 confirmed reactive samples) and a specificity of $97.6 \%(1,050$ out of 1,076$)$.

Although the sensitivity is not very high, the testing approach nevertheless reduces the operational impact of addressing a minimal risk from travellers and offers improvement over deferral by testing all former residents of endemic areas. Despite the current international regulatory requirements, the current national policies have "evolved" through a series of additions and revisions to the guidelines as concerns and issues arose in each country. This has led to relatively high variability in donor selection criteria currently used internationally. In non-endemic countries, the prevention of imported malaria is addressed by two distinct strategies. The donors must be deferred for long enough after returning from an endemic region, thus allowing the potentially infected donors to develop symptoms or resolve the infection. Alternatively, they are tested for signs of malaria disease after a shorter deferral period [17]. The following limitations of our study should be pointed out, however. Samples that were negative in the initial screening ELISA were only confirmed negative by the index ELISA, but not at the reference laboratory. The confirmation of negative test results is currently not required in current routine strategy used in the blood centres and was therefore not actively pursued. Although considered rather unlikely, it cannot be excluded that the different initial ELISAs may miss samples that are reactive with the malaria ELISA and IFAT 
using total $P$. falciparum antigens as is used at the reference laboratory. In addition, since the EUROIMMUN ELISA also contains $P$. knowlesi protein antigens, it could be one possible explication that non-confirmed positive results from the EUROIMMUN ELISA may arise form from P. knowlesi-specific antibodies that do not crossreact with the $P$. vivax and $P$. falciparum antigens. Information on the direct clinical or the geographical history of the blood donors is mostly lacking. A direct detection of the pathogen by either molecular testing or microscopy is considered not feasible due to low-level parasitaemias in the blood donor samples. For these reasons, it is difficult to investigate possible reasons for the observed discrepancies between the two test systems.

\section{Conclusion}

This study shows the necessity of selective screening of blood donations for anti-Plasmodium antibodies in blood transfusion centres in Germany and Switzerland, two non-endemic regions. Four Plasmodium IgG-positive blood donations were identified using both the screening and index tests and confirmed with the reference laboratory tests. It is possible these donations were from donors with an active malaria infection and thus posed a potential source of TTM. On the other hand, the reactive samples could also have originated from donors with a persistent high antibody titre from a previously treated malaria case. As the national guidelines request, these donations were rejected due to safety reasons.

Of course, with the used approach of a serological test based on the questionnaire there will never exist a 100\% safety in terms of Plasmodium spp. An advantage of routine screening for anti-Plasmodium antibodies of blood donations with increased risk is that a negative screening result leads to a reduced deferral period of the corresponding donor. In this way, the profitability of the blood banks is maximized without compromising blood safety.
The comparison of the new EUROIMMUN ELISA and the established CAPTIA ${ }^{\mathrm{TM}}$ Malaria EIA (Trinity Biotech) and Malaria EIA (BioRad) used for routine blood donor screening in two laboratory centres reveals that all three ELISAs are equally suitable for anti-Plasmodium antibody screening in blood banks.

\section{Acknowledgements}

We would like to thank Dr. Christoph Schaefer for his support and assistance in relation to the collaboration of the three parties.

\section{Statement of Ethics}

The testing of donors for Plasmodium spp. is a routine scheme used in both implicated blood transfusion services. In addition, the donors agree to use parts of their donation for quality control and quality assurance. The data and parts of the donation are only used in anonymous or coded form.

\section{Conflict of Interest Statement}

J.M.K. and J.M.W. are employed by EUROIMMUN Medizinische Labordiagnostika, which has filed a patent application pertaining to a method for the detection of antibodies to Plasmodium. The remaining authors have no competing financial interests.

\section{Funding Sources}

The authors did not receive any external funding.

\section{Author Contributions}

Study design and supervision: C.N., J.D., J.M.W, J.M.K. Performed the experiments: C.T., T.V., H.P.M., B.N. Analysed the data: P.G. Wrote the manuscript: C.N., P.G., J.M.K. Edited the manuscript: C.N., C.T., J.D., T.V., H.P.M., B.N., J.M.K., J.M.W., P.G.

\section{References}

1 Ahmed MA, Cox-Singh J. Plasmodium knowlesi - an emerging pathogen. ISBT Sci Ser. 2015;10(Suppl 1):134-40.

2 Anthony CN, Lau YL, Sum JS, Fong MY, Ariffin $\mathrm{H}$, Zaw WL, et al. Malaysian child infected with Plasmodium vivax via blood transfusion: a case report. Malar J. 2013;12:308.

3 Chiodini PL, Hartley S, Hewitt PE, Barbara JA, Lalloo K, Bligh J, et al. Evaluation of a malaria antibody ELISA and its value in reducing potential wastage of red cell donations from blood donors exposed to malaria, with a note on a case of transfusion-transmitted malaria. Vox Sang. 1997;73(3):143-8.
4 Frey-Wettstein M, Maier A, Markwalder K, Munch U. A case of transfusion transmitted malaria in Switzerland. Swiss Med Wkly. 2001;131(21-22):320.

5 Garraud O, Assal A, Pelletier B, Danic B, Kerleguer A, David B, et al. Overview of revised measures to prevent malaria transmission by blood transfusion in France. Vox Sang. 2008; 95(3):226-31.

6 Romi R, Sabatinelli G, Majori G. Malaria epidemiological situation in Italy and evaluation of malaria incidence in Italian travelers. J Travel Med. 2001;8(1):6-11.
7 Scuracchio P, Vieira SD, Dourado DA, Bueno LM, Colella R, Ramos-Sanchez EM, et al. Transfusion-transmitted malaria: case report of asymptomatic donor harboring Plasmodium malariae. Rev Inst Med Trop Sao Paulo. 2011;53(1):55-9.

8 Slinger R, Giulivi A, Bodie-Collins M, Hindieh F, John RS, Sher G, et al. Transfusion-transmitted malaria in Canada. CMAJ. 2001;164(3):377-9.

9 Pathak S, Chandrashekhar M. Transfusion transmittable infections - seroprevalence among blood donors in a tertiary care hospital of Delhi. Asian J Transfus Sci. 2013;7(2):116-8. 
10 Kitchen AD, Barbara JA, Hewitt PE. Documented cases of post-transfusion malaria occurring in England: a review in relation to current and proposed donor-selection guidelines. Vox Sang. 2005;89(2):77-80.

11 Kitchen AD, Chiodini PL. Malaria and blood transfusion. Vox Sang. 2006;90(2):77-84.

12 Kitchen AD, Lowe PH, Lalloo K, Chiodini PL. Evaluation of a malarial antibody assay for use in the screening of blood and tissue products for clinical use. Vox Sang. 2004;87(3):150-5.

13 Niederhauser C, Gottschalk J, Tinguely C. Selective testing of at-risk blood donors for Trypanosoma cruzi and Plasmodium spp. in Switzerland. Transfus Med Hemother. 2016; 43(3):169-76

14 Reesink HW, Panzer S, Wendel S, Levi JE, Ullum H, Ekblom-Kullberg S, et al. The use of malaria antibody tests in the prevention of transfusion-transmitted malaria. Vox Sang. 2010;98(3 Pt 2):468-78
15 Seed CR, Kee G, Wong T, Law M, Ismay S. Assessing the safety and efficacy of a testbased, targeted donor screening strategy to minimize transfusion transmitted malaria. Vox Sang. 2010;98(3 Pt 1):e182-92.

16 Blumel J, Burger R, Drosten C, Groner A, Gurtler L, Heiden M, et al. Malaria. Transfus Med Hemother. 2008;35(2):122-34.

17 Seed CR, Kitchen A, Davis TM. The current status and potential role of laboratory testing to prevent transfusion-transmitted malaria. Transfus Med Rev. 2005;19(3):229-40.

18 She RC, Rawlins ML, Mohl R, Perkins SL, Hill HR, Litwin CM. Comparison of immunofluorescence antibody testing and two enzyme immunoassays in the serologic diagnosis of malaria. J Travel Med. 2007;14(2):105-11.

19 Medina Costa R, de Sousa KP, Atouguia J, Tavira LT, Silva MS. Prevalence and level of antibodies anti-Plasmodium spp. in travellers with clinical history of imported malaria. J Parasitol Res. 2013;2013:247273.
20 Swiss Transfusion Swiss Red Cross (SRC) Guidelines: Kapitel 11 (A): Spendenanalytik: Infektionsmarker. Bern: Swiss Transfusion SRC; 2017 [updated July 1, 2017]. Available from: https://sbsc-bsd.ch/dokuman2/de-de/ bsd/vorschriften/kapitel.aspx (accessed March 28, 2018).

21 Grande R, Petrini G, Silvani I, Simoneschi B, Marconi M, Torresani E. Immunological testing for malaria and blood donor deferral: the experience of the Ca' Granda Polyclinic Hospital in Milan. Blood Transfus. 2011;9(2): 162-6.

22 O'Brien SF, Delage G, Seed CR, Pillonel J, Fabra CC, Davison K, et al. The epidemiology of imported malaria and transfusion policy in 5 nonendemic countries. Transfus Med Rev. 2015;29(3):162-71. 